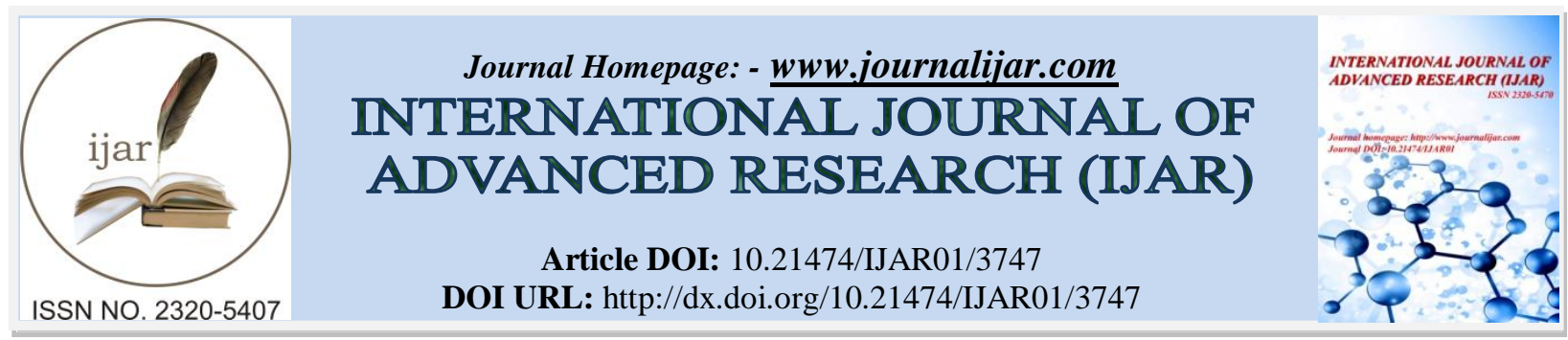

RESEARCH ARTICLE

\title{
SURVEY ON CONTRIBUTIONS TOWARDS LIVE FACE RECOGNITION IN VIDEO STREAMING.
}

\author{
Yashraj Manshani, Shreya Nair, Sazia Shaikh, Suraj Rana, Aditi Kalia and D.A. Phalke.
}

D.Y. Patil College of Engineering, Akurdi, Pune.

\section{Manuscript Info}

Manuscript History

Received: 16 January 2017

Final Accepted: 02 February 2017

Published: March 2017

Key words:-

Image attributes,Face

recognition,Templates,Compression,

Content analysis, Video content

extraction, Image processing, Temporal

segmentation, Scene segmentation.

\begin{abstract}
Image detection to identify the targeted image and extract its contents in any of the live videos which are being streamed. Every particular image of a layman contains several unique attributes for Example, the space between the eyebrows or a different type of nose. The proposed project looks into recognizing this special features and detecting them through the videos being processed in of the environment. This was attributed to the ability of artificial intelligence management, as a computer technology, to generate, process, and analyze large volume of data at a time, compared to when humans are used, to help managers in quick decision making because results of the analysis are available in time.
\end{abstract}

Copy Right, IJAR, 2017,. All rights reserved.

\section{Introduction:-}

With the advancement in technology and more and more invention being done in the field of computer technology, the risk of theft and destruction has been increased to vast levels. A security system for the protection of all kinds of destructive things round the world has become one of the major necessity.This kind of a system would be creating help to not on only the field of technology but also in vehicle monitoring, theft and illegal activity investigation. The approach can also be used in detecting murders and crimes happening around.

A self-recognizing system that detects image in live video streaming is being studied. Every human face is different, every face is gifted with certain attributes that is one's own identity and this makes each face different from other. This personal identity can be used as a tool to detect and verify different people round the ground.The basic requirement for the work are: Images, for representing the frame data, a numerical matrix whose values can be colours, colour map entries, etc.Colour Maps, which map entries into a colour space, allowing an additional indexation level.Image Display Converters and Image IO Handlers, that convert images in the specific formats of theplatforms and vice-versa

The approach seeks to store the unique feature of the image in its database. Further, the extraction of this feature is verified through Eigen- Face algorithm. Here whenever the system detects an image, it crops the unique feature from the face and creates a similar possible and identical image of the face in its image dataset.so the next time the same face occurs in front of the face it detects the image and extracts the contents about the image from the video streaming. This indirectly detects and monitors

\section{Related work:-}

The idea for extracting the content of information is the Accessibility Problem. A problem that is even more relevant for dynamic multimedia data, which also have to be searched and retrieved. While content extraction techniques are 
reasonably developed for text, video data still is essentially opaque. Its richness and complexity suggests that there is a long way to go in extracting video features, and the implementation of more suitable and Effective processing procedures are an important goal to be achieved [1]. The increase in the diversity and availability of electronic information led to additional processing requirements, in order to retrieve relevant and useful data: the accessibility problem. This problem is even more relevant for audio visual information, where huge amounts of data have to be searched, indexed and processed. Most of the solutions for this type of problems point towards a common need: to extract relevant information features for a given content domain [2]. A process which underlies two difficult tasks: deciding what is relevant and extracting it. In fact, while content extraction techniques are reasonably developed for text, video data still is essentially opaque. Despite its obvious advantages as a communication medium, the lack of suitable processing and communication supporting platforms has delayed its introduction in a generalized way. This situation is changing and new video based applications are being developed. In our research group, we are tools for indexing video archives for later reuse, a system for content analysis of TV news, and hyper video systems where hyperlinks are established based on content identification in different video streams. These applications greatly rely on efficient computational support, combining powerful image analysis and processing [3].Each of these concepts is represented by a $(\mathrm{C}++)$ class and integrated in a systematic hierarchy. Tools for data processing are applied to the described data modelling classes, and also modelled as a hierarchy of classes [4]: The Image OPs, these operators represent functions which are applied to image regions and extract "single-image" or sequential content features. A shot object is a discrete sequence of images with a set of temporal attributes such as frame rate and durationand represents a video segment [5]. A shot sequence object groups several shots using some semantic criteria. Views, are used to visualize and browse shots and shot sequences.

The approach in this study is to construct dynamic templates in a bottom-up fashion using information on problem types and associated discourse structure [6]. The classification algorithm uses different cropping styles to detect the $3 \mathrm{D}$ image in the video streaming.

Face recognition has been a sough after problem of biometrics and it has a variety of applications in modern life. The problems of face recognition attract researchersworking in biometrics, pattern- recognition and computer vision. Several face recognition algorithms are also used in many different applications apart from biometrics, such as video compressions, indexing's etc. [7]. They can also be used to classify multimedia content, to allow fast and efficient searching for material that is of interest to the user. An efficient face recognition system can be of great help in forensic sciences, identification for law enforcement, surveillance authentication for banking and security system, and giving preferential access to authorised users i.e. access control for secured areas etc. [8]. The problem of face recognition has gained even more importance after the recent increase in the terrorism related incidents.

\section{Background: Image Detection:-}

This section introduces image detection and presents a list of common performance objectives and the techniques used to achieve them. Moreover, a brief overview of the evolution of image processing until the appearance of the PCE-based architecture is included. Finally, the limitations of current solutions are described, in order to identify how system can be future improved.

A.In this paper, it proposes to improve MPW with the aimof sampling a smaller number of PWs without any loss indetection rate and false positive rate. In addition, we solve the problem of determining the number of PWs in each stage. We call the proposed algorithm iPW does not need to generate a large number of PWs at the initial iteration (stage), which greatly reduces the detection time. Even if the initial PWs do not contain any object, iPW can detect the objects at next stages.2) MPW unnecessarily draws too many PWs around the positive windows whereas iPW avoids generating there redundant PWs by using the information of both rejectedand accepted PWs.3) To use MPW, one has to empirically set the number of PWs in each stage whereas this is not a problem becauseIPW generates a single PW in each iteration (stage).4) IPW fully makes use of the information of rejected negativePWs while MPW almost completely depends on theaccepted positive PWs. Rejected negative PWs are usedto directly suppress the PD around these windows andat the same time indirectly enhance the PD beyond thewindows. In this sense, I PW is rejection-oriented whileMPW is acceptance-oriented.5) In MPW, the uniform distribution is used in the initialization and plays an unimportant role in the later stages so that it can be omitted. In iPW, a dented uniform distribution is used to play an important role for sampling useful PWs by rejecting background.IPW fully makes use of the information of rejected negative PWs while MPW almost completely depends on theaccepted positive PWs. Rejected negative PWs are usedto directly suppress the PD around these windows andat the same time indirectly enhance the PD beyond thewindows. In this sense, I PW is rejection-oriented whileMPW is acceptance-oriented.5) 
In MPW, the uniform distribution is used in the initialization stage and play unimportant role in theater stages so that it can be omitted. In iPW, a dented uniform distribution is used to play an important role for sampling useful PWs by rejecting background(i.e., no object) regions.6) iPW utilizes dented Gaussian distribution while MPWutilizes full Gaussian distribution for sampling PWs. Byusing dented Gaussian distribution, iPW avoids drawingmany unnecessary PWs around the object and object-likeregions.7)

B. It provides a comprehensive review on the recent advances in face anti-spoofing. While most previous works on face spoofing detection are based on analyzing only the luminance (i.e. gray-scale) information of the face images, we introduce a novel and appealing approach using color texture analysis and demonstrate that the Chroma component can be very useful in discriminating fake faces from genuine ones. We exploit the joint color-texture information from theluminance and the chrominance channels by computinglow-level features using different descriptors extractedfrom different colorspaces. We investigate how welldifferentcolor spaces and descriptors can be used for describing the intrinsic disparities in the color texture between genuine faces andfake ones. We also perform afusion study to analyze the complementarity of the different descriptors and different colorspaces. We conduct extensive experimental analysis on the three latest and most challenging spoofing databases using their predefined publicly well-defined experimental evaluation protocols ensuring the reproducibility of the results anda fair comparison against the state of the art. We provide an extensive comparative analysis againstthe state-of-the-art face spoofing detection techniques andshow that our proposed approach outperforms all existingmethods on two databases and achieves very competitiveperformance on the third databaseUnlike most of the methods proposed in the literature, our proposed approach is able to achieve stable performance across all the three benchmark datasets. Furthermore, in our inter-database evaluation, the facial color texture representation showed promising generalization capabilities, thus suggesting that color texture can be more stable in unknown conditions compared to its gray-scale counterparts.

Research onnon-intrusive software-based face spoofingdetection has mainly been focusing on analyzing grayscaleimages and hence discarding the color information whichcan be a vital visual cue for discriminating fake faces fromgenuine ones. In a very recent work, Wen et al. [6] proposedcolor quality based features that describe the chromaticdegradation and the lack of color diversity of recaptured faceimages. However, the actual local variations in color textureinformation was not exploited for face spoofing detection

There are many ways to perform face scrambling. Forexample, scrambling can be done simply by masking orcartooning. However, this kind of scrambling will simplylose the facial information, and hence subsequent facerecognition or verification becomes unsuccessful in this case.Especially for security reasons, it is obviously not a goodchoice to really erase human faces from surveillance videos.In comparison, the Arnold transform as a basic step in many encryption algorithms, is a kind of recoverable scrambling method. Scrambled faces can be unscrambledby several manual tries.

C.Moving object detection plays an important rolein many video processing applications, such asobject tracking, categorization, identification, and videocondensation. It often serves as pre-processing for higher levelvideo analyses and its performance directly affects the performance of the subsequent applications. For object tracking, if moving object is detected as two or two moving objects are detected as one, the tracking result may be incorrect. For object categorization, incomplete or adhesive detection of moving objects may lead to wrong categorization, and it is the same case for object identification. For video condensation, object tracking is also an indispensable part. It's not the desired result if the head and legs of one person appear at different time in the condensed video. Ideally, a detection method should detect each moving object separately without breaking. Backgroundmodelling is indispensable for moving object detection in many cases and many works have been done in this research area. In early works, the background model was constructed for each pixel independently. In , a single Gaussian model was used to model the value variations of each pixel, and the parameters of the Gaussian model were updated recursively with an adaptive filter. It is robust in modelling the static background, but is sensitive to dynamic background variations. To address these problems, the mixture of Gaussian models (Moggs) was proposed in . In addition, a series of variants were proposed to improve the performance of background modelling. However, the Gaussian model-based methods are all based on the assumption that the pixel intensity follows a Gaussian distribution, which is not always correct. In contrast, a nonparametric method was proposed in, where each pixel is directly modelled with a probability density function without any assumption on the distribution of the pixel values. Since all the possible pixel values are modelled into the probability density function, dynamic background modelling problem can be handled and illumination change can be adapted progressively. In [6], a Bayesian decision rule for 
background and foreground classification was constructed based on different feature selections for static and moving backgrounds, and is able to deal with dynamic background problems better than MoG. In [7] and [8], background pixel values are quantized into a set of codebooks to form the background model which is efficient in memory and speed.

D. Given a training dataset, faces are forwarded to the training procedure. The offline procedure then learns its semantic salience map. Following this, the database is scrambled and the feature space is reconstructed by multiplying salient feature according to their semantic salience weights. Random sampling is then applied to select features sparsely to construct as many kernels as is allowed, and discriminant analysis is used to learn a kernel subspace for each kernelAfter a scrambled facial image is input as a test, the input isprojected into each kernel subspace, and the distance to eachtraining sample is computed. The decision procedure is basedon the combination of all kernel subspaces/It is noted that we can have unscrambled images (mainly forstatistic salience learning) in the offline training because offline training is carried out centrally with authorities'/business supervisors' permission and will not undermine users' privacy. Privacy protection is mainly an issue with distribution over the internet. In this scheme, the training procedure can be carried out offline. The online verification then becomes purely a datadriven process. In the test procedure, all test images. we have identified a new challenge in scrambled face recognition originated from the need for biometric verification in emerging IoT applications, and developed a salience-aware face recognition scheme that can work with chaotic patterns in the scrambled domain. In our method, we conjectured that scrambled facial recognition could generate a new problem in which "many manifolds" need to be discovered for discriminating these chaotic signals, and we proposed a new ensemble approach - Many-Kernel Random Discriminant Analysis (MK-RDA) for scrambled face recognition. We also incorporated a salience-aware strategy into the proposed ensemble method to handle chaotic facial patterns in the scrambled domain, where random selection of features is biased towards semantic components via salience modelling. In our experiments, the proposed MK-RDA was tested rigorously on three standard human face datasets. The experimental results successfully validated that the proposed scheme can effectively handle chaotic signals and drastically improve the recognition accuracy, making our method a promising candidate for emerging IoT applications One of the most important tasks for video analysis is to specify a unit set, in which the video temporalsequence may be organized.

The different video transitions are important for video content identificationand for the definition of the semantics of the video language, making their detection one of the primarygoals to be achieved. The basic assumption of the transition detection procedures is that the video segmentsare spatially and temporally continues, and thus the boundary images must suffer significant content changes. Changes, which depend on the transition type and can be measured. The original problem is reduced to the search of suitable difference quantification metrics, whose maximums identify, with great probability, the transition temporal locations

Goals:-

Once installed, test whether or not it works by firing up a python session. If there are no any errors then, move furtherNext step is the cascading the image (the cascade is just a Xml file that contains the data to detect faces. Read the image and convert it to greyscale. Many operations in Open $\mathrm{Cv}$ are done in grayscale. This is detection of the face that comes in front of camera. Once the face detection is done, next part to be done is its recognition in such a way that it stores the value of each faces, so that the next time the face appears on the screen it is able to name and retrieve data by its own. For this Eigen-face Algorithm is used, theEigen values of each face is stored under the database.Prepare a training set of face images. The pictures constituting the training set should have been taken under the same lighting conditions, and must be normalized to have the eyes and mouths aligned across all images. Subtract the mean. The average image a has to be calculated and then subtracted from each original image in Calculate the eigenvectors and eigenvalues of the covariance matrix $\mathbf{S}$. Choose the principal components. Sort the eigenvalues in descending order and arrange eigenvectors accordingly. The number of principal components $\mathrm{k}$ is determined arbitrarily by setting a threshold $\varepsilon$ on the total variance. These Eigen faces can now be used to represent both existing and new faces.

\section{Identified Challenges:-}

Extensive experiments on the three latest and most challenging spoofing databases (the CASIA FASD, the ReplayAttackDatabase and MSU MFSD) showed excellent results. On theCASIA FASD and the MSU MFSD, the proposed facial colour texture representation based on the combination CoLBP and LPQ features computed over HSV and YCbCr colour spaces outperformed the state of the art, while very competitive results were achieved on the Replay-Attack Database. More importantly, the proposed approach was able to achieve stable performance 
across all three benchmark datasets unlike most of the methods proposed in the literature. Furthermore, in our interdatabase evaluation, the facial colour texture representation showed promising generalization capabilities, thus suggesting that colour texture seems to be more stable in unknown conditions compared to its gray-scale counterparts. To be consistent with many previous studies, it is worth opting that face normalization or the limits of the face bounding box were not optimized in our experiments.

\section{Identified Analysis:-}

The system contains four modules:

Image detection, Image recognition, Image classification and content extraction. Image detection is the basic step towards identification of the image here the face in front of the screen is identified and a cropping structure (dialog box) appears over the detected area. The next step is to recognize the unique feature of the face the system recognized the basic pixels and creates the base feature of the face. Further a similar possible image of the face is created using this base feature and the mage is classified under various folders The live streaming data of the previously stored is compared and the contents from the image is detected and conclusions are made

\section{Experimental Setup:-}

Following explain the basic experimentalsetup of the system:

Accept the image from the live video streams

Classify it and crop appropriate features

Identify function using Eigen-face algorithm

Generate equations (with the help of Eigen values)

Solve Equations

Display Content

\section{Summary:-}

present a multi-stage investigation system that can be use in various fields of cyber security systems. The crimes and various threats can be easily solved through recognizing images in the pictures as well as live video streams ensuring a safety towards destructive world.

\section{References:-}

1. Wright, J. and Yi Ma and Mairal, J. and Sapiro, G. and Huang, T.S. and Shuicheng Yan, \Robust Face Recognition via Sparse Representation," IEEE Conference on Computer Vision and Pattern Recognition. CVPR 2009. pp.597 -604, 2009

2. Yiming Yang and Xin Liu, "A re-examination oftextcategorization methods" in Proceedings of the 22ndannualinternational ACM SIGIR conference on Researchanddevelopment in information retrieval ACM,1999.

3. D. Nadeau and S. Sekine, "A Survey of NamedEntityRecognition and Classification", LinguisticaeInvestigations, vol.30,2007.

4. Ronan Collobert, Jason Weston, Leon Bottou,MichaelKarlen, Koray Kavukcuoglu, Pavel Kuksa, "NaturalLanguageProcessing (Almost) from Scratch" in Journal ofMachineLearning Research 12 (2011) 2493-2537 in2011.

5. Y. Li, K. Xu, Q. Yan, Y. Li, and R. H. Deng, "Understanding OSN-basedfacial disclosure against face authentication systems," in Proc. 9th ACMSymp. Inf., Comput. Commun. Secur. (ASIA CCS), 2014, pp413-424.

6. J. Li, Y. Wang, T. Tan, and A. K. Jain, "Live face detection based on the analysis of Fourier spectra," Proc. SPIE, vol. 5404, pp. 29

7. X. Tan, Y. Li, J. Liu, and L. Jiang, "Face liveness detection from a single image with sparse low rank bilinear discriminative model," in Proc. 11thEur. Conf. Comput. Vis., VI (ECCV), 2010, pp. 504-517.

8. Z. Zhang, J. Yan, S. Liu, Z. Lei, D. Yi, and S. Z. Li, "A face ant spoofing database with diverse attacks," in Proc. 5th IAPR Int. Conf Biometrics (ICB), Mar./Apr. 2012, pp. 26-31.

9. J. Globally and S. Marcel, "Face anti-spoofing based on general image quality assessment," in Proc. IAPR/IEEE Int. Conf. PatternRecognit. (ICPR), Aug. 2014, pp. 1173-1178.

10. D. Wen, H. Han, and A. K. Jain, "Face spoof detection with image distortion analysis," IEEE Trans. Inf. Forensics Security, vol. 10, no. 4,pp. 746-761, Apr. 2015.

11. J. Bai, T.-T. Ng, X. Gao, and Y.-Q. Shi, "Is physics-based liveness detection truly possible with a single image?" in Proc. IEEE Int. Symp.Circuits Syst. (ISCAS), May/Jun. 2010, pp. 3425-3428.

12. J. Määttä, A. Hadid, and M. Pietikäinen, "Face spoofing detection fromsingle images using micro-texture 
analysis," in Proc. Int. Joint Conf.Biometrics (IJCB), Oct. 2011, pp. 1-7.

13. J. Maatta, A. Hadid, and M. Pietikäinen, "Face spoofing detection fromsingle images using texture and local shape analysis," IET Biometrics. 1, no. 1, pp. 3-10, Mar. 2012.

14. J. Yang, Z. Lei, S. Liao, and S. Z. Li, "Face liveness detection with component dependent descriptor," in Proc. IAPR Int. Conf. Biometrics (ICB), Jun. 2013, pp. 1-6.

15. T. de Freitas Pereira et al., "Face liveness detection using dynamic texture," EURASIP J. Image Video Process., vol. 2014, no. 1, pp. 1-15, Dec. 2014.

16. Pinto, H. Pedrini, W. R. Schwartz, and A. Rocha, "Face spoofingdetection through visual codebooks of spectral temporal cubes," IEEETrans. Image Process., vol. 24, no. 12, pp. 4726-4740, Dec. 2015.

17. K. Kollreider, H. Fronthaler, and J. Bigun, "Non-intrusive liveness detection by face images," Image Vis. Comput., vol. 27, no. 3, pp. 233-244, 2009.

18. Anjos and S. Marcel, "Counter-measures to photo attacks in face recognition: A public database and a baseline," in Proc. IAPR IEEE Int.

19. Joint Conf. Biometrics (IJCB), Oct. 2011, pp. 1-7.

20. J. Yan, Z. Zhang, Z. Lei, D. Yi, and S. Z. Li, "Face liveness detection exploring multiple scenic clues," in Proc. 12th Int. Conf. ControlAutom. Robot. Vis. (ICARCV), Dec. 2012, pp. 188-193.

21. N. Kose and J.-L. Dugelay, "Countermeasure for the protection office recognition systems against mask attacks," in Proc. 10th Int.Conf. Autom. Face Gesture Recognit., Shanghai, China, Apr. 2013, pp. 1-6.

22. T. de Freitas Pereira, A. Anjos, J. M. De Martino, and S. Marcel, "Canface anti-spoofing countermeasures work in a real-world scenario?" pinprick. Int. Conf. Biometrics (ICB), Jun. 2013, pp. 1-8.

23. Chingovska and A. R. dos Anjos, "On the use of client identity information for face ant spoofing," IEEE Trans. Inf. Forensics Security, vol. 10, no. 4, pp. 787-796, Apr. 2015.

24. Yang, Z. Lei, D. Yi, and S. Z. Li, "Person-specific face ant spoofingwith subject domain adaptation," IEEE Trns. Inf. Forensics. 